\title{
Fertilization outcome, embryo development and birth after unstimulated IVM
}

\author{
Adriana Bos-Mikich • Marcelo Ferreira • \\ Marcos Höher • Gerta Frantz • Norma Oliveira • \\ Caroline G. Dutra $\cdot$ Nilo Frantz
}

Received: 4 May 2010 /Accepted: 23 September 2010 /Published online: 6 October 2010

(C) Springer Science+Business Media, LLC 2010

\begin{abstract}
Background/Purposes A common observation in oocyte in vitro maturation (IVM) cycles is poor embryo quality. However, no study was dedicated to assess zygote and early cleavage embryo quality in IVM cycles. The objective of this study is to analyze fertilization outcome, embryo development and the resulting pregnancy and births in unstimulated IVM cycles.

Methods IVM oocytes were collected $36 \mathrm{~h}$ post hCG and matured in vitro for 28-30 h. All oocytes were inseminated by ICSI. Resulting zygotes and embryos were assessed on day-1, 2 and 3, when transfers were made.

Results The overall oocyte maturation and fertilization rates were $63 \%$ and $62 \%$, respectively. Abnormal fertilization rate was $1.7 \%$. Ninety five and $14.6 \%$ of the $2 \mathrm{Pn}$ zygotes reached the 2-cell and 8 -cell stage at day-2 and day-3, respectively. Embryo quality assessment on day-3 at transfer revealed that only $9 \%$ of the embryos were of very good quality. Most embryos showed developmental delay. An average of 3.29 embryos were transferred per patient resulting in implantation and clinical gestation rates of $16 \%$ and $32 \%$, respectively. Overall 14 healthy babies were born and there is one ongoing pregnancy.
\end{abstract}

Capsule In vitro maturation of human oocytes results in reasonably high rates of pregnancy and birth being particularlly indicated for PCO or PCOS patients.

\section{A. Bos-Mikich $(\square)$}

Department of Morphological Sciences, ICBS,

Universidade Federal do Rio Grande do Sul,

Porto Alegre, RS, Brazil, CEP: 90.050-170

e-mail: adriana.bosmikich@gmail.com

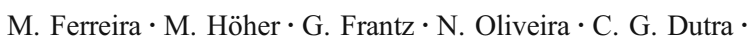

N. Frantz

Nilo Frantz Research and Human Reproduction Centre,

Porto Alegre, RS, Brazil, CEP: 90.480-003
Conclusion Results show a significant rate of abnormal fertilization and poor embryo quality after IVM, which is reflected in a higher than average number of embryos being transferred. However, pregnancy, implantation and birth rates are reasonably high and allow us to consider IVM a valuable approach for the treatment of infertility in PCO or PCOS patients.

Keywords IVM · Embryo quality · Implantation · Pregnancy $\cdot$ Births

\section{Introduction}

The development of new biotechnologies for assisted reproduction (AR) is always desired. The introduction of in vitro maturation in human reproduction represented a valuable choice of protocol, when dealing with patients at risk of ovarian hyperstimulation using exogenous gonadotrophins for classical AR technologies. Since the first report of human oocyte IVM [6], the methodology has been refined and considerable progress has been achieved in terms of oocyte maturation and pregnancy rates.

IVM reported success rates vary considerably among services $[1,2,4,8,11,17,18,19,21]$. It is acknowledged that the methodology for IVM of oocytes collected from unstimulated ovaries and embryo development is still not ideal [12]. A common observation in IVM cycles is the poor embryo quality and the high number of cleavage stage embryos being transferred leading to lower implantation rates, when compared with classic stimulated cycles. We reported on the first IVM pregnancy and birth in Brazil [7]. Since our first attempts with the methodology, we noticed that IVM zygotes have a higher incidence of abnormal fertilization and embryos have a poorer in vitro perfor- 
Table 1 Fertilization and embryo development outcome of 34 IVM cycles

\begin{tabular}{ll} 
Variable & \\
\hline No. of patients & 34 \\
Age $(y)($ mean \pm SD) & $28.88 \pm 4.67$ \\
No. Immature oocytes cultured (mean) & $556(16.3)$ \\
No. of MII oocytes (maturation rate) & $350(63 \%)$ \\
No. oocytes fertilized & $218(62 \%)$ \\
No. abnormal fertilization $(1 \mathrm{Pn} / \geq 3 \mathrm{PN})($ mean) & $58(1.7)$ \\
No. cleaving 2Pn embryos & $208(95 \%)$ \\
No. of 8C-embryos at Day-3 (mean) & $32(0.94)$ \\
No. embryos transferred Day-3 (mean) & 3.29 \\
Pregnancy rate per transfer $(\%)$ & $11 / 34(32)$ \\
Multiple pregnancies $(\%)$ & $5 / 11(45)$ \\
Implantation rate $(\%)$ & $18 / 112(16)$ \\
Births & $10 / 11$ \\
Sex ratio (male/female) & $6 / 8$ \\
\hline
\end{tabular}

mance, compared with stimulated cycles. Also, there are suggestions that birth rates are lower in IVM cycles, which would undermine its application as a valuable infertility treatment approach.

Here we report on the fertilization outcome, embryo quality and development, implantation, pregnancy and birth rates after in vitro maturation of oocytes in unstimulated cycles.

\section{Material \& methods}

\section{Patient selection}

This is a retrospective analysis of 34 IVM cycles that occurred between January 2007 and December 2009. We analyzed data from 27 cycles of patients with polycystic ovarian syndrome (PCOS) and seven cycles of isolated polycystic ovaries (PCO) [14].

IVM patients had a baseline scan performed between days 2 and 4 of either a natural menstrual cycle or, if amenorrhoeic, after withdraw bleed induced by progesterone. This assessment recorded the number of follicles, endometrial thickness, ovarian volume and the presence of cysts. The same assessment was repeated between days 6 and 8 and timing of hCG was based on endometrial thickness $(\geq 7 \mathrm{~mm})$.

Oocyte retrieval, fertilization, embryo culture and transfer

Immature oocyte collection was performed $36 \mathrm{~h}$ post-hCG injection using a 17- gauge aspiration needle with a reduced aspiration pressure of $80 \mathrm{mmHg}$. Cumulus-oocytecomplexes were matured in IVM Medium (Medicult) supplemented with $75 \mathrm{mIU} / \mathrm{ml}$ of FSH (Gonal-F, Serono) and $0.1 \mathrm{IU} \backslash \mathrm{ml}$ of hCG (Chorimon, Meizler) for 28 to $30 \mathrm{~h}$. After maturation, oocytes were denuded of cumulus cells by exposure to hyaluronidase and pipetting.

Insemination of MII oocytes was by ICSI using the partner's spermatozoa. Injected oocytes were cultured in ECM medium (Early Cleavage Medium, Irvine Scientific) in $5.5 \% \mathrm{CO}_{2}$ in air, at $37^{\circ} \mathrm{C}$ under oil. Fertilization check was performed $18 \mathrm{~h}$ post-ICSI for both groups of patients. Zygotes were further cultured in ECM medium till embryo transfer on day-3, when embryo quality was assessed [20], approximately $72 \mathrm{~h}$ post-insemination. All very good and good quality embryos (grades $1 \& 2$ ) that were not transferred were cryopreserved.

Patients received the best scoring embryos for transfer. All women received $6 \mathrm{mg}$ of oestradiol valerate (Estrofem, Medley) for endometrial preparation, starting on the day of oocyte collection. Luteal support was provided by $800 \mathrm{mg}$ of natural micronized progesterone (Evocanil, Zodiac) starting on day 1 and continued until 12 weeks of gestation together with the oestradiol valerate. Clinical pregnancy was diagnosed by the presence of a gestational sac, 4 weeks after oocyte pick up.

\section{Results}

Table 1 shows the age of the patients, maturation and fertilization rates, embryo development, pregnancy and implantation rates for the 34 IVM cycles here analyzed.

In vitro maturation rate and fertilization rates were $63 \%$ and $62 \%$ respectively. The frequency of abnormally fertilized oocytes ( $1 \mathrm{Pn}, \geq 3 \mathrm{Pn}$ ) was $1.7 \%$, mainly due to second polar body retention. Nearly all normally fertilized zygotes reached the 2-cell stage on day-2, but only $14.6 \%$ of these embryos were at the 8-cell stage at transfer on day-3. In average, more than 3 embryos were transferred for each patient. Following embryo transfer, clinical pregnancy and implantation rates were 32 and $16 \%$, respectively. There were five multiplets gestations, out of which four were twins (two became singletons) and one a quadruplet. There was one late abortion, nine healthy babies delivered (eight girls and six boys) and one ongoing gestation of a baby girl.

Table 2 Quality of IVM embryos at transfer on Day-3

\begin{tabular}{lr}
\hline Embryo grade & IVM \\
\hline G 1 & $09(08 \%)$ \\
G 2 & $27(24 \%)$ \\
G 3 & $64(57 \%)$ \\
G4 & $12(11 \%)$ \\
Total D3 (TE) & 112 \\
\hline
\end{tabular}


Table 2 details the quality of day- 3 embryos at transfer. Only $9 \%$ of all day-3 embryos were of very good quality and they were transferred to five different patients, all of which got pregnant. The majority of the embryos $(68 \%)$ was of poor (G3) or very poor (G4) quality particularly due to retarded development.

\section{Discussion}

The data here presented show that embryo quality and development to day-3 are impaired in IVM cycles with oocytes from PCO or PCOS patients. However, implantation and pregnancy rates are reasonable and close to those usually attained in classical stimulated ICSI cycles. Birth and sex ratio of the babies delivered after IVM do not present any significant difference from babies born after other assisted reproduction technologies or natural conception.

There is a limited number of reports describing the embryological aspects of IVM [4, 8, 17, 18] particularly their developmental competence. This is a critical point in IVM procedures, as there are concerns that this approach leads to relatively high rates of developmental incompetent oocytes, which may affect gestation and subsequent birth rates.

Son et al. [18] reported that better pregnancy rate is obtained in hCG-primed IVM cycles, in which in vivo matured oocytes were collected compared with IVM cycles without any in vivo matured oocytes. However, the authors have a rather low implantation rate for both groups of patients and that may suggest that embryo quality was similar between the two groups. The lower embryo quality in that study may also be inferred from the elevated mean number of embryos transferred for both groups of patients.

Earlier studies comparing IVF and IVM claimed that implantation and pregnancy rates were lower after IVM in PCO and PCOS patients $[5,10]$. However, these data have been questioned after a meta-analysis showed that there are no randomized controlled trials upon which one can base a conclusion on the use of IVM or conventional IVF for patients with PCOS [16]. On the other hand, a recent report [21] and our own experience show that implantation and pregnancy rates around 16 and $32-36 \%$, respectively, after IVM are much superior to the first reports and close to results attained after classical IVF/ICSI cycles. This observation, together with the fact that we had five multiple gestations suggests that, although day-3 embryo quality seems to be impaired after IVM, their developmental potential is not affected and an IVM-own embryo scoring system should be proposed to compensate for their developmental delay. In doing so, a lower number of embryos should be transferred to avoid the occurrence of multiple gestations and its unwanted outcomes.
We found that after IVM, $1.7 \%$ of the fertilized oocytes present abnormal pronuclear formation, particularly $3 \mathrm{Pn}$ due to second polar body retention. This frequency is lower than that reported $(2.5 \%)$ after insemination by IVF or ICSI of more than 5000 human oocytes in conventional IVF/ ICSI cycles [13]. However, it is higher than the observed rate of polyploidy after conventional ICSI in our service. The reason for these discrepancies is not clear, however, it can be assumed that failure of second polar body extrusion after ICSI in IVM cycles is related to cytoskeletal abnormalities, possibly caused by manipulation or incomplete cytoplasmic maturation.

One main difference between IVM and classical IVF cycles is that IVM oocytes spend a more prolonged period in vitro before insemination. There are evidences that prolonged in vitro culture is related to an increased rate of monozygotic twinning in classical IVF/ICSI cycles [15]. However, we did not observe any monozygotic twinning among the multiplet gestations here described. These data agree with previous reports $[3,9]$ and suggests that IVM itself does not increase the rate of monozygotic twinning.

Except for the quadruplet pregnancy, all others were uneventfull and 14 healthy babies were delivered, which demonstrates that IVM is a reliable alternative approach for PCO and PCOS patients that need assisted reproduction technologies for achieving a pregnancy.

In conclusion, despite the occurrence of abnormal fertilization and lower embryo quality, progresses in terms of implantation and pregnancy rates and the birth of healthy babies, together with its lower costs and avoidance of ovarian hyperstimulation lead us to believe that IVM is a clinically valuable technology in human AR, with clear application for young PCO and PCOS patients.

\section{References}

1. Cha KY, Chung HM, Lee DR, Kwon H, Chung MK, Park LS, et al. Obstetric outcome of patients with polycystic ovary syndrome treated by in vitro maturation and in vitro fertilization-embryo transfer. Fertil Steril. 2005;83:1461-5.

2. Chian RC, Buckett WM, Tulandi T, Tan SL. Prospective randomized study of human chorionic gonadotrophin priming before immature oocyte retrieval from unstimulated women with polycystic ovarian syndrome. Hum Reprod. 2000;15:165-70.

3. Chian RC. In-vitro maturation of immature oocytes for infertile women with PCOS. RBM Online. 2004;8:547-52.

4. Child TJ, Abdul-Jalil AK, Gulekli B, Tan SL. In vitro maturation and fertilization of oocytes from unstimulated normal ovaries, and women with polycystic ovary syndrome. Fertil Steril. 2001;76:936-42.

5. Child TJ, Phillips SJ, Abdul-Jalil AK, Gulekli B, Tan SL. A comparison of in vitro maturation and in vitro fertilization for women with polycystic ovaries. Obstet Gynecol. 2002;100:66570. 
6. Edwards RG. Maturation in vitro of human ovarian oocytes. Lancet. 1965;2:926-9.

7. Frantz N, Bos-Mikich A, Frantz G, Höher M, Ferreira M. Gravidez após a maturação in vitro de oócitos obtidos sem estimulação hormonal em paciente com ovários policísticos. JBRA. 2008;12:45-7.

8. Ge HS, Huang XF, Zhang W, Zhao JZ, Lin JJ, Zhou W. Exposure to human chorionic gonadotrophin during in vitro maturation does not improve the maturation rate and developmental potential of immature oocytes from patients with polycystic ovary syndrome. Fertil Steril. 2008;89:98-103.

9. Kanaya H, Murata Y, Oku H, Hashimoto S, Morimoto Y, Murata $\mathrm{T}$, et al. Successful monozygotic twin delivery following in vitro maturation of oocytes retrieved from a woman with polycystic ovary syndrome: case report. Hum Reprod. 2006;21:1777-80.

10. Khashavi Z, Karimzadeh MA. Comparison of unstimulated in vitro maturation and stimulated in vitro fertilization in women with polycystic ovarian syndrome. J Res Med Sci. 2004;6:260-3.

11. Le Du, Kadoch IJ, Bourcigaux N, Doumerc S, Bourrier MC, Chevalier N, et al. In vitro oocyte maturation for the treatment of infertility associated with polycystic ovarian syndrome: the French experience. Hum Reprod. 2005;20:420-4.

12. Mikkelsen AL, Smith S, Lindenberg S. Possible factors affecting the development of oocytes in in vitro maturation. Hum Reprod. 2000;15 Suppl 5:11-7.

13. Porter R, Han T, Tucker MJ, Graham J, Liebermann J, Sills ES. Estimation of second polar body retention rate after conventional insemination and intracytoplasmic sperm injection: in vitro observations from more than 5000 human oocytes. J Ass Reprod Genet. 2003;20.

14. Rotterdam ESHRE $\backslash A S R A M-2004$ Sponsored PCOS Consenus Workshop Group. Revised 2003 consensus on diagnotic-criteria and long-term health risks related to polycystic ovary syndrome. Fertil Steril. 2003;81:19-25.

15. Sills ES, Tucker MJ, Palermo GD. Assisted reproductive technologies and monozygous twins: implications for future study and clinical practice. Twin Res. 2000;3:217-23.

16. Siristadilis CS, Maheshwari A, Bhattacharya S. In vitro maturation in subfertile women with polycystic ovarian syndrome undergoing assisted reproduction. Cochrane Database Sys Rev. 2009;21:CD006606.

17. Son WY, Lee SY, Lim JH. Fertilization, cleavage and blastocyst development according to the maturation timing of oocytes in in vitro maturation cycles. Hum Reprod. 2005;20:3204-7.

18. Son W-Y, Chung Lee S, Lim J-H. Comparison of in-vitro maturation cyles with and without in vivo matured oocytes retrieved. RBM Online. 2008;17:59-67.

19. Sönderström-Anttila V, Mäkinen S, Tuuri T, Suikkari AM. Favorable pregnancy results with insemination of in vitro matured oocytes from unstimulated patients. Hum Reprod. 2005;20:1534-40.

20. Veeck L. Atlas of the Human oocyte and early conceptus, vol.2. Baltimore: Williams \& Wilkins; 1999.

21. Zhao J-Z, Zhou W, Zhang W, Ge HS, Huang XF, Lin JJ. In vitro maturation and fertilization of oocytes from unstimulated ovaries in infertile women with polycystic ovary syndrome. Fertil Steril. 2009;91:2568-71. 